\title{
Coexistence of identical synchronization, antiphase synchronization and inverse full state hybrid projective synchronization in different dimensional fractional-order chaotic systems
}

\author{
Adel Ouannas ${ }^{1}$, Xiong Wang ${ }^{2}$, Viet-Thanh Pham ${ }^{3 *}$, Giuseppe Grassi ${ }^{4}$ and Toufik Ziar ${ }^{5}$
}

${ }^{*}$ Correspondence:

phamvietthanh@tdt.edu.vn

${ }^{3}$ Modeling Evolutionary Algorithms

Simulation and Artificial Intelligent,

Faculty of Electrical \& Electronics

Engineering, Ton Duc Thang

University, Ho Chi Minh City,

Vietnam

Full list of author information is

available at the end of the article

\section{Springer}

\begin{abstract}
The topic related to the coexistence of different synchronization types between fractional-order chaotic systems is almost unexplored in the literature. Referring to commensurate and incommensurate fractional systems, this paper presents a new approach to rigorously study the coexistence of some synchronization types between nonidentical systems characterized by different dimensions and different orders. In particular, the paper shows that identical synchronization (IS), antiphase synchronization (AS), and inverse full state hybrid projective synchronization (IFSHPS) coexist when synchronizing a three-dimensional master system with a fourth-dimensional slave system. The approach, which can be applied to a wide class of chaotic/hyperchaotic fractional-order systems in the master-slave configuration, is based on two new theorems involving the fractional Lyapunov method and stability theory of linear fractional systems. Two examples are provided to highlight the capability of the conceived method. In particular, referring to commensurate systems, the coexistence of IS, AS, and IFSHPS is successfully achieved between the chaotic three-dimensional Rössler system of order 2.7 and the hyperchaotic four-dimensional Chen system of order 3.84. Finally, referring to incommensurate systems, the coexistence of IS, AS, and IFSHPS is successfully achieved between the chaotic three-dimensional Lü system of order 2.955 and the hyperchaotic four-dimensional Lorenz system of order 3.86 .
\end{abstract}

MSC: $34 \mathrm{H} 10 ; 26 \mathrm{~A} 33 ; 34 \mathrm{~A} 08$

Keywords: coexistence of synchronization types; fractional-order systems; chaos synchronization; commensurate and incommensurate systems; nonidentical systems

\section{Introduction}

By starting from the milestone by Pecora and Carroll [1], over the last years, great efforts have been devoted to the study of chaos synchronization in dynamical systems described by integer-order differential equations and difference equations [2]. Given two systems in the master-slave configuration, the objective in chaos synchronization is to make the response system variables synchronized in time with the corresponding drive system vari-

(c) The Author(s) 2018. This article is distributed under the terms of the Creative Commons Attribution 4.0 International License (http://creativecommons.org/licenses/by/4.0/), which permits unrestricted use, distribution, and reproduction in any medium, provided you give appropriate credit to the original author(s) and the source, provide a link to the Creative Commons license, and indicate if changes were made. 
ables. At the beginning, Pecora and Carroll introduced the concept of complete (identical) synchronization (IS), but, year after year, different types of synchronization have been proposed in the literature, for continuous- and discrete-time systems [3-10]. For example, in projective synchronization (PS) the response system variables are scaled replicas of the drive system variables [11]. When the scaling factor is ' -1 ', antiphase synchronization $(A S)$ is obtained. On the other hand, when the scaling factor is different for each drive system variable, full state hybrid projective synchronization (FSHPS) is achieved [12]. Recently, in $[13,14]$ a synchronization scheme has been presented, where each drive system state synchronizes with a linear combination of response system states. Since drive system states and response system states have been inverted in $[13,14]$ with respect to the FSHPS, the scheme has been called inverse full state hybrid projective synchronization (IFSHPS). Additionally, recent papers have investigated the coexistence of several synchronization types when synchronizing two chaotic systems both in integer-order differential systems and discrete-time systems [15-17]. In particular, the approach developed in [15] has illustrated a rigorous study to prove the coexistence of some synchronization types between discrete-time chaotic (hyperchaotic) systems.

Besides integer-order systems, attention has been recently focused on systems described by fractional-order differential equations $[18,19]$. Researches in the literature have shown that fractional-order systems, as generalizations of well-known integer-order systems, are characterized by chaotic dynamics [20,21]. These systems include the fractional Lorenz system, the fractional Chua system, the fractional Rössler system, the fractional Chen system, and the fractional Lü system [22-26]. Specifically, researches have shown that chaos is achievable when the system order is less than 3, whereas hyperchaos can be obtained when the system order is less than 4 . Referring to synchronization, studies have shown that chaotic fractional-order systems can also be synchronized [27]. However, differently from integer-order systems, few synchronization types have been introduced for fractional-order systems. Moreover, most of the approaches are related to the synchronization of identical fractional-order systems. Very few methods for synchronizing nonidentical fractional-order chaotic systems have been illustrated [28-30]. Additionally, the topic related to the coexistence of different synchronization types between fractionalorder systems is almost unexplored [31, 32].

Based on these considerations, this paper presents a new approach to rigorously study the coexistence of some synchronization types between fractional-order systems characterized by different dimensions and different orders. In particular, the paper shows that identical synchronization (IS), antiphase synchronization (AS), and inverse full state hybrid projective synchronization (IFSHPS) coexist in the synchronization of a three-dimensional master system with a four-dimensional slave system. The approach presents the remarkable feature of being both rigorous and applicable to a wide class of commensurate and incommensurate fractional-order systems of different dimensions and different orders. It is worth noting that the proposed approach is more general than those illustrated in [31, 32] because, among other things, it guarantees the coexistence of three different synchronization types (instead of only two types, as in [31,32]). This increased complexity provides a deeper insight into the synchronization phenomena between systems described by fractional differential equations.

The paper is organized as follows. In Section 2, the basic notions on fractional derivatives and on the stability of fractional systems are given. In Section 3, the meaning of co- 
existence of IS, AS, and IFSHPS in fractional-order systems is illustrated. In Section 4, by exploiting fractional Lyapunov stability theory the coexistence of IS, AS, and IFSHPS between two commensurate fractional-order systems of different dimensions is proved. Additionally, by using the stability theory of fractional linear systems, in Section 5, the coexistence of IS, AS, and IFSHPS between two incommensurate fractional-order systems of different dimensions is illustrated. Referring to numerical examples, at first the coexistence of IS, AS, and IFSHPS between the chaotic fractional-order commensurate Rössler system and the hyperchaotic fractional-order commensurate Chen system is successfully achieved. Note that the Rössler system is a three-dimensional system of order 2.7, whereas the Chen system is a four-dimensional system of order 3.84. An additional numerical example illustrates the coexistence of IS, AS and IFSHPS between the chaotic fractional-order the incommensurate Lü system and the hyperchaotic fractional-order incommensurate Lorenz system. Note that the Lü system is a three-dimensional system of order 2.955, whereas the Lorenz system is a four-dimensional system of order 3.86. All the numerical examples prove the capability of the proposed approach in successfully achieving the coexistence of $I S, A S$, and IFSHPS between chaotic and hyperchaotic systems of different dimensions for both commensurate and incommensurate fractional-order systems. Finally, in Section 6, the advantages and the novelty of the conceived approach with respect to those existing in the literature are discussed in detail.

\section{Basic concepts}

In the following, some basic concepts on fractional derivatives and on the stability properties of fractional linear/nonlinear systems are briefly illustrated.

Definition 1 ([33]) The Caputo fractional derivative is defined as follows:

$$
D_{t}^{p} x(t)=J^{m-p} x^{m}(t) \quad \text { with } 0<p \leq 1,
$$

where $m=[p]$ (i.e., $m$ is the first integer that is not less than $p$ ), $x^{m}$ is the $m$ th-order derivative in the usual sense, and $J^{q}(q>0)$ is the $q$ th-order Riemann-Liouville integral operator defined by

$$
J^{q} y(t)=\frac{1}{\Gamma(q)} \int_{0}^{t}(t-\tau)^{q-1} y(\tau) d \tau
$$

where $\Gamma$ denotes the gamma function [34-36].

Now we state a well-known theorem on the stability of fractional linear systems.

Theorem 1 ([37]) Let

$$
D_{t}^{p_{i}} x_{i}(t)=\sum_{j=1}^{n} k_{i j} x_{i}(t), \quad i=1,2, \ldots, n,
$$

be a fractional linear system where $p_{i}(i=1,2, \ldots, n)$ are rational numbers between 0 and 1 , whereas $D_{t}^{p_{i}}$ is the Caputo fractional derivative of order $p_{i}$. Assume that $p_{i}=\frac{\alpha_{i}}{\beta_{i}},\left(\alpha_{i}, \beta_{i}\right)=1$, $\alpha_{i}, \beta_{i} \in \mathbb{N}$. Let $M$ be the least common multiple of the denominators $\beta_{i}$ of $p_{i}$. If $p_{i}$ are different 
rational numbers between 0 and 1 , then system (3) is asymptotically stable, provided that all the roots $\lambda$ of the equation

$$
\operatorname{det}\left(\operatorname{diag}\left(\lambda^{M p_{1}}, \lambda^{M p_{2}}, \ldots, \lambda^{M p_{n}}\right)-K\right)=0
$$

satisfy the condition $|\arg (\lambda)|>\frac{\pi}{2 M}$, where $K=\left(k_{i j}\right)_{n \times n}$.

Now we report a theorem on fractional nonlinear systems.

Theorem $2([38,39])$ Consider the fractional nonlinear system

$$
D_{t}^{p} X(t)=F(X(t))
$$

where $X(t) \in \mathbb{R}^{n}, 0<p<1, D_{t}^{p}$ is the Caputo fractional derivative of order $p$, and $F: \mathbb{R}^{n} \rightarrow$ $\mathbb{R}^{n}$. If there exists a positive definite Lyapunov function $V(X(t))$ such that $D_{t}^{p} V(X(t))<0$ for all $t>0$, then the trivial solution of system (5) is asymptotically stable.

Starting from previous theorem, we can give the following lemma.

Lemma 1 ([40]) We have:

$$
\frac{1}{2} D_{t}^{p}\left(X^{T}(t) X(t)\right) \leq X^{T}(t) D_{t}^{p}(X(t)) .
$$

\section{Problem formulation}

Referring to synchronization, let

$$
D_{t}^{p_{i}} x_{i}(t)=\sum_{j=1}^{3} a_{i j} x_{j}(t)+f_{i}(X(t)), \quad i=1,2,3,
$$

be the master system, where $X(t)=\left(x_{1}(t), x_{2}(t), x_{3}(t)\right)^{T}$ is the state vector, $\left(a_{i j}\right) \in \mathbb{R}^{3 \times 3}$ are constant parameters, $f_{i}: \mathbb{R}^{3} \rightarrow \mathbb{R}$ are nonlinear functions, and $D_{t}^{p_{i}}$ is the Caputo fractional derivative of order $p_{i}(i=1,2,3)$ with $0<p_{i}<1$. Moreover, let

$$
D_{t}^{q_{i}} y_{i}(t)=g_{i}(Y(t))+u_{i}, \quad i=1,2, \ldots, 4,
$$

be the slave system, where $Y(t)=\left(y_{1}(t), y_{2}(t), \ldots, y_{4}(t)\right)^{T}$ is the state vector, $g_{i}: \mathbb{R}^{4} \rightarrow \mathbb{R}$ are nonlinear functions, $D_{t}^{p_{i}}$ is the Caputo fractional derivative of order $q_{i}\left(0<q_{i}<1\right)$, and $u_{i}$ are controllers to be designed $(i=1,2, \ldots, 4)$.

Based on the master-slave synchronizing system described by (7)-(8), we can give the following definition of coexistence of different synchronization types.

Definition 2 Identical synchronization (IS), antiphase synchronization (AS), and inverse full state hybrid projective synchronization (IFSHPS) coexist in the synchronization of the master system (7) and the slave system (8) if there exist controllers $u_{i}(1 \leq i \leq 4)$ and real numbers $\left(\alpha_{31}, \alpha_{32}, \alpha_{33}, \alpha_{34}\right)$ such that the synchronization errors

$$
e_{1}(t)=x_{1}(t)-y_{1}(t)
$$




$$
\begin{aligned}
& e_{2}(t)=x_{2}(t)-\left(-y_{2}(t)\right), \quad \text { and } \\
& e_{3}(t)=x_{3}(t)-\sum_{j=1}^{4} \alpha_{3 j} y_{j}(t)
\end{aligned}
$$

satisfy the condition $\lim _{t \rightarrow+\infty} e_{i}(t)=0, i=1,2,3$.

\section{Coexistence of IS, AS, and IFSHPS for commensurate systems}

In this section, we focus ourselves on the coexistence of IS, AS, and IFSHPS for commensurate chaotic (hyperchaotic) systems. First, we prove a new theorem. Afterward, we illustrate in detail an application involving the chaotic fractional-order Rössler system (as a master system) and the hyperchaotic fractional-order Chen system (as a slave system).

We assume that $q_{1}=q_{2}=q_{3}=q_{4}=q$. The Caputo fractional derivative of order $q$ of the error system (9) can be derived as

$$
\begin{aligned}
& D_{t}^{q} e_{1}(t)=D_{t}^{q} x_{1}(t)-g_{1}(Y(t))-u_{1}, \\
& D_{t}^{q} e_{2}(t)=D_{t}^{q} x_{2}(t)+g_{2}(Y(t))+u_{2}, \\
& D_{t}^{q} e_{3}(t)=D_{t}^{q} x_{3}(t)-\sum_{j=1}^{4} \alpha_{3 j} g_{j}(Y(t))-\sum_{j=1}^{4} \alpha_{3 j} u_{j} .
\end{aligned}
$$

On the other hand, the error system (10) can be rewritten in compact form as

$$
D_{t}^{q} e(t)=A e(t)+R-\alpha \times U
$$

where

$$
\begin{aligned}
& e(t)=\left(e_{1}(t), e_{2}(t), e_{3}(t)\right)^{T}, \quad A=\left(a_{i j}\right)_{3 \times 3}, \quad \alpha=\left(\begin{array}{cccc}
1 & 0 & 0 & 0 \\
0 & -1 & 0 & 0 \\
\alpha_{31} & \alpha_{32} & \alpha_{33} & \alpha_{34}
\end{array}\right), \\
& R=-A e(t)+\left(\begin{array}{c}
D_{t}^{q} x_{1}(t)-g_{1}(Y(t)) \\
D_{t}^{q} x_{2}(t)+g_{2}(Y(t)) \\
D_{t}^{q} x_{3}(t)-\sum_{j=1}^{4} \alpha_{3 j} g_{j}(Y(t))
\end{array}\right), \quad \text { and } U=\left(u_{1}, u_{2}, u_{3}, u_{4}\right)^{T}
\end{aligned}
$$

Now we can prove a new theorem.

Theorem 3 Identical synchronization (IS), antiphase synchronization (AS), and inverse full state hybrid projective synchronization (IFSHPS) coexist in the synchronization of the master system (7) and the slave system (8) if the control law is selected as follows:

$$
\begin{aligned}
& \left(u_{1}, u_{2}, u_{3}\right)^{T}=\boldsymbol{J}^{-1} \times(R+C e(t)), \\
& u_{4}=0,
\end{aligned}
$$

where $\mathbf{J}^{-1}$ is the inverse matrix of

$$
\boldsymbol{J}=\left(\begin{array}{ccc}
1 & 0 & 0 \\
0 & -1 & 0 \\
\alpha_{31} & \alpha_{32} & \alpha_{33}
\end{array}\right)
$$


and $C \in \mathbb{R}^{3 \times 3}$ is a suitable control matrix such that $(A-C)$ is a negative definite matrix.

Proof By exploiting (13) the error system (11) becomes

$$
D_{t}^{q} e(t)=A e(t)+R-\boldsymbol{J} \times\left(u_{1}, u_{2}, u_{3}\right)^{T} .
$$

By applying the control law proposed in (12) to (14), the resulting error dynamics are described by

$$
D_{t}^{q} e(t)=(A-C) e(t) .
$$

If the candidate Lyapunov function is chosen as $V(e(t))=\frac{1}{2} e^{T}(t) e(t)$, then the time Caputo fractional derivative of order $q$ along the trajectory of system (14) is described as

$$
D_{t}^{q} V(e(t))=\frac{1}{2} D_{t}^{q}\left(e^{T}(t) e(t)\right) .
$$

By applying Lemma 1 to equation (16) it follows that

$$
D_{t}^{q} V(e(t)) \leq e^{T}(t) D_{t}^{q} e(t)
$$

By (15) it results in

$$
D_{t}^{q} V(e(t)) \leq e^{T}(t)(A-C) e(t)<0
$$

From Theorem 2 it can be readily deduced that the zero solution of system (15) is globally asymptotically stable. Consequently, IS, AS, and IFSHPS coexist in the synchronization of the master system (7) and the slave system (8).

Now, we give an example to illustrate how the previous theoretical framework can be effectively applied. In particular, the chaotic fractional-order commensurate Rössler system is considered as the master system [41]

$$
\left\{\begin{array}{l}
D_{t}^{p} x_{1}=-\left(x_{2}-x_{3}\right), \\
D_{t}^{p} x_{2}=x_{1}+\alpha x_{2}, \\
D_{t}^{p} x_{3}=x_{3}\left(x_{1}-\gamma\right)+\beta
\end{array}\right.
$$

As shown in [39], a chaotic attractor is found in (19) when $(\alpha, \beta, \gamma)=(0.4,0.2,10)$ and $p=0.9$ (see Figure 1). By taking these parameters, the linear part of the master system (19), according to equation (7), is given by

$$
A=\left(\begin{array}{ccc}
0 & -1 & 1 \\
1 & 0.4 & 0 \\
0 & 0 & -10
\end{array}\right) .
$$



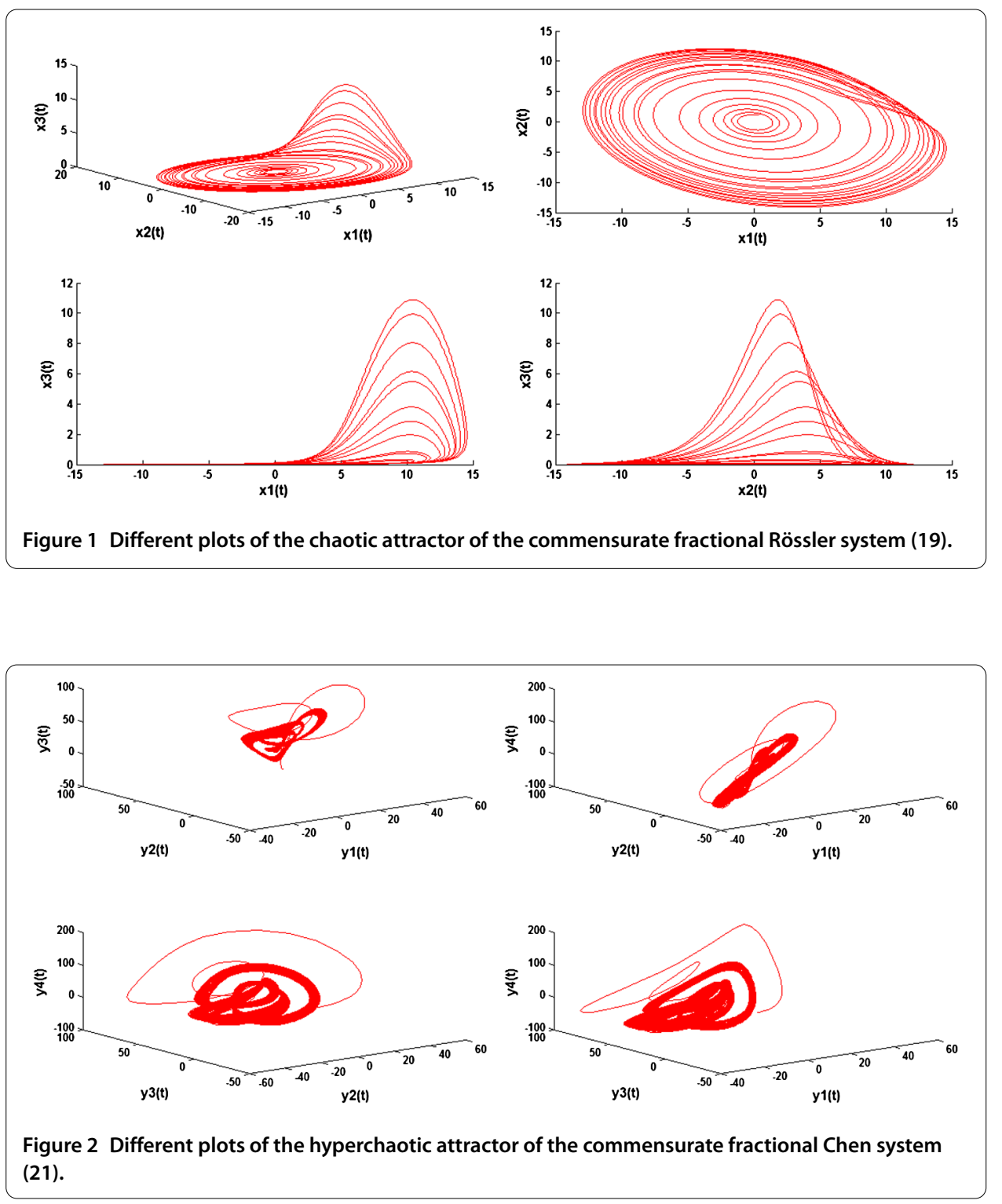

The hyperchaotic fractional-order commensurate Chen system is considered as the slave system [42]

$$
\left\{\begin{array}{l}
D_{t}^{q} y_{1}=a\left(y_{2}-y_{1}\right)+y_{4}+u_{1}, \\
D_{t}^{q} y_{2}=d y_{1}+c y_{2}-y_{1} y_{3}+u_{2}, \\
D_{t}^{q} y_{3}=-b y_{3}+y_{1} y_{2}+u_{3}, \\
D_{t}^{q} y_{4}=k y_{4}+y_{2} y_{3}+u_{4}
\end{array}\right.
$$

where $U=\left(u_{1}, u_{2}, u_{3}, u_{4}\right)^{T}$ is the vector controller. According to [40], system (21) exhibits hyperchaotic behavior when $q=0.96,(a, b, c, d, k)=(35,3,12,7,0.5)$, and $\left(u_{1}, u_{2}, u_{3}, u_{4}\right)=$ $(0,0,0,0)$. Different plots of the hyperchaotic attractor are reported in Figure 2. 
By selecting the scaling parameters

$$
\alpha=\left(\alpha_{i j}\right)_{3 \times 4}=\left(\begin{array}{cccc}
1 & 0 & 0 & 0 \\
0 & -1 & 0 & 0 \\
2 & 3 & 4 & 5
\end{array}\right)
$$

the error system between the master system (19) and the slave system (21) becomes

$$
\begin{aligned}
& e_{1}=x_{1}-y_{1}, \\
& e_{2}=x_{2}-\left(-y_{2}\right), \\
& e_{3}=x_{3}-\left(2 y_{1}+3 y_{2}+4 y_{3}+5 y_{4}\right) .
\end{aligned}
$$

Successively, we derive the following matrices:

$$
\begin{aligned}
& \boldsymbol{J}=\left(\begin{array}{ccc}
1 & 0 & 0 \\
0 & -1 & 0 \\
2 & 3 & 4
\end{array}\right), \\
& \boldsymbol{J}^{-1}=\left(\begin{array}{ccc}
1 & 0 & 0 \\
0 & -1 & 0 \\
\frac{-1}{2} & \frac{3}{4} & \frac{1}{4}
\end{array}\right) .
\end{aligned}
$$

According to Theorem 3, there exists a control matrix $C \in \mathbb{R}^{3 \times 3}$ such that $I S$, $A S$, and IFSHPS coexist in the synchronization of the master system (19) and the slave system (21). For example, by selecting the control matrix

$$
C=\left(\begin{array}{ccc}
1 & -1 & -1 \\
1 & 4.4 & 0 \\
0 & 0 & 0
\end{array}\right)
$$

it can be readily verified that $(A-C)$ is a negative definite matrix. By exploiting matrix (25) it follows from Theorem 3 that the control inputs can be written in the form (12)-(13) as

$$
\begin{aligned}
u_{1}= & e_{1}+D^{0.96} x_{1}-35\left(y_{2}-y_{1}\right)-y_{4}, \\
u_{2}= & -4 e_{2}-D^{0.96} x_{2}-7 y_{1}+y_{1} y_{3}-12 y_{2}, \\
u_{3}= & -\frac{1}{2} e_{1}-3 e_{2}+2.5 e_{3}+D^{0.96}\left(-\frac{1}{2} x_{1}-\frac{3}{4} x_{2}+\frac{1}{4} x_{3}\right)-35 y_{1}-18 y_{2}+3 y_{3} \\
& -\frac{2.5}{4} y_{4}+\frac{3}{2} y_{1} y_{3}-y_{1} y_{2}-\frac{5}{4} y_{2} y_{3}, \\
u_{4}= & 0 .
\end{aligned}
$$

Since all the conditions of Theorem 3 are satisfied, it follows that IS, AS, and IFSHPS coexist in the synchronization of the master system (19) and the slave system (21). The error 


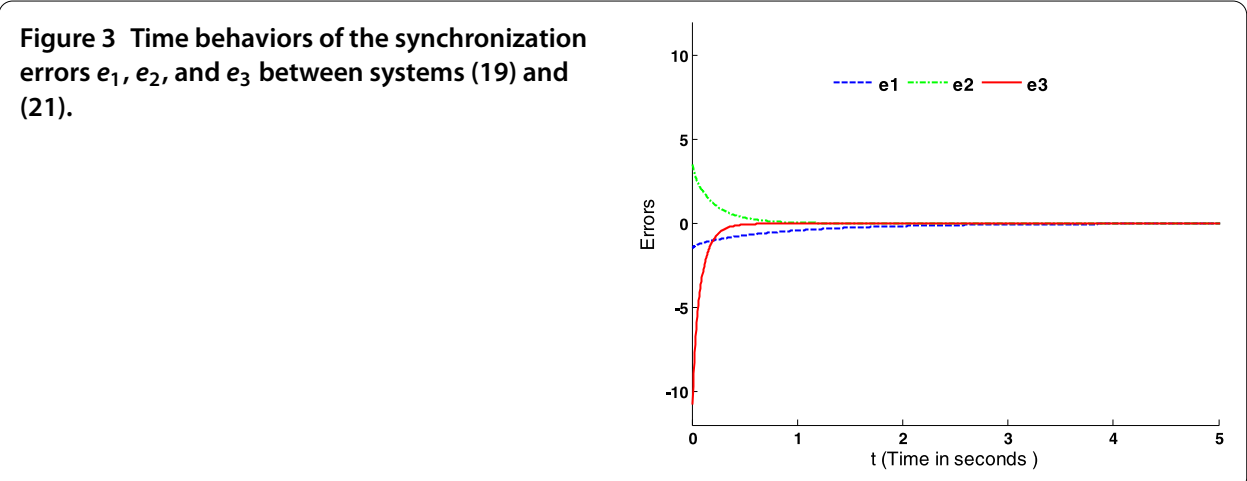

dynamics are described as follows:

$$
\left\{\begin{array}{l}
D^{0.96} e_{1}=-e_{1}, \\
D^{0.96} e_{2}=-4 e_{2}, \\
D^{0.96} e_{3}=-10 e_{3} .
\end{array}\right.
$$

Besides the theoretical results illustrated, to show by numerical simulations that the coexistence is effectively achieved, the fractional Euler integration method has been used to solve the systems (19) and (21) with the control inputs described in (27). The initial values of the master system and the slave system are $\left[x_{1}(0), x_{2}(0), x_{3}(0)\right]=[0.5,1.5,0.1]$ and $\left[y_{1}(0), y_{2}(0), y_{3}(0), y_{4}(0)\right]=[2,2,-1,1]$, respectively. Based on (23), the initial states of the error system are $\left[e_{1}(0), e_{2}(0), e_{3}(0)\right]=[-1.5,3.5,-10.8]$. The time behaviors of the errors $e_{i}(i=1,2,3)$ are shown in Figure 3, clearly indicating that synchronization is effectively achieved between systems (19) and (21). Specifically, by considering the errors in (23), it can be concluded identical synchronization (IS), anti-phase synchronization (AS) and inverse full state hybrid projective synchronization (IFSHPS) coexist when synchronizing the chaotic fractional-order commensurate Rössler system (19) and the hyperchaotic fractional-order commensurate Chen system (21).

\section{Coexistence of $I S, A S$, and IFSHPS for incommensurate systems}

In this section, we analyze the coexistence of IS, AS, and IFSHPS for incommensurate chaotic (hyperchaotic) systems. A new theorem, tailored for synchronizing incommensurate systems, is first proved. Successively, an example involving the chaotic fractionalorder Lü system (as a master system) and the hyperchaotic fractional-order Lorenz system (as a slave system) is described in detail.

We first assume that $q_{1} \neq q_{2} \neq q_{3}$. Then, the Caputo fractional derivative for order $q_{i}$, $1 \leq i \leq 3$, of the error system (10) can be derived as

$$
\begin{aligned}
& D_{t}^{q_{1}} e_{1}(t)=D_{t}^{q_{1}} x_{1}(t)-g_{1}(Y(t))-u_{1}, \\
& D_{t}^{q_{2}} e_{2}(t)=D_{t}^{q_{2}} x_{2}(t)+g_{2}(Y(t))+u_{2}, \\
& D_{t}^{q_{3}} e_{3}(t)=D_{t}^{q_{3}} x_{3}(t)-D_{t}^{q_{3}}\left(\sum_{\substack{j=1 \\
j \neq 3}}^{4} \alpha_{3 j} y_{j}(t)\right)-\alpha_{33} g_{3}(Y(t))-\alpha_{33} u_{3} .
\end{aligned}
$$


To synchronize the master system (7) and the slave system (8), we assume that $u_{4}=0$ and $\alpha_{33} \neq 0$. Then, the error system (29) can be rewritten in compact form as

$$
D_{t}^{\hat{q}} e(t)=A e(t)+T-\operatorname{diag}\left(1,-1, \alpha_{33}\right) \times \hat{U},
$$

where

$$
\begin{aligned}
& D_{t}^{\hat{q}} e(t)=\left(D_{t}^{q_{1}} e_{1}(t), D_{t}^{q_{2}} e_{2}(t), D_{t}^{q_{3}} e_{3}(t)\right)^{T}, \\
& A=\left(a_{i j}\right)_{3 \times 3}, \\
& \hat{U}=\left(u_{1}, u_{2}, u_{3}\right)^{T}, \\
& T=-A e(t)+\left(\begin{array}{c}
D_{t}^{q_{1}} x_{1}(t)-g_{1}(Y(t)) \\
D_{t}^{q_{2}} x_{2}(t)+g_{2}(Y(t)) \\
D_{t}^{q_{3}} x_{3}(t)-D_{t}^{q_{3}}\left(\sum_{\substack{j=1 \\
j \neq 3}}^{4} \alpha_{3 j} y_{j}(t)\right)-\alpha_{33} g_{3}(Y(t))
\end{array}\right) .
\end{aligned}
$$

Now we can give a new theorem for synchronizing incommensurate systems.

Theorem 4 Given the error system (29) between the master system (7) and the slave system (8), identical synchronization (IS), antiphase synchronization (AS), and inverse full state hybrid projective synchronization (IFSHPS) coexist if the following control law is taken:

$$
\hat{U}=\operatorname{diag}\left(1,-1, \frac{1}{\alpha_{33}}\right) \times(T+L e(t)),
$$

provided that the feedback gain matrix $L \in \mathbb{R}^{3 \times 3}$ is selected so that all roots $\lambda$ of the equation

$$
\operatorname{det}\left(\operatorname{diag}\left(\lambda^{M q_{1}}, \lambda^{M q_{2}}, \lambda^{M q_{3}}\right)+L-A\right)=0
$$

satisfy the condition $|\arg (\lambda)|>\frac{\pi}{2 M}$, where $M$ is the least common multiple of the denominators of $q_{i}, i=1,2,3$.

Proof By applying the control law (32) to the error system of the form (30), the resulting error dynamics are described by

$$
D_{t}^{\hat{q}}=(A-L) e(t) .
$$

If the feedback gain matrix $L$ is chosen so that all roots $\lambda$ of equation (33) satisfy the condition $|\arg (\lambda)|>\frac{\pi}{2 M}$, then by Theorem 1 we can conclude that the zero solution of the error system (34) is globally asymptotically stable. Consequently, IS, AS, and IFSHPS coexist between systems (7) and (8).

Now we give an example to illustrate how the previous theoretical framework for incommensurate systems can be effectively applied. In particular, as a master system, we consider the chaotic fractional-order incommensurate Lü system [43]

$$
\left\{\begin{array}{l}
D_{t}^{p_{1}} x_{1}=\alpha\left(x_{2}-x_{1}\right), \\
D_{t}^{p_{2}} x_{2}=-x_{1} x_{3}+\gamma x_{2}, \\
D_{t}^{p_{3}} x_{3}=x_{1} x_{2}-\beta x_{3} .
\end{array}\right.
$$



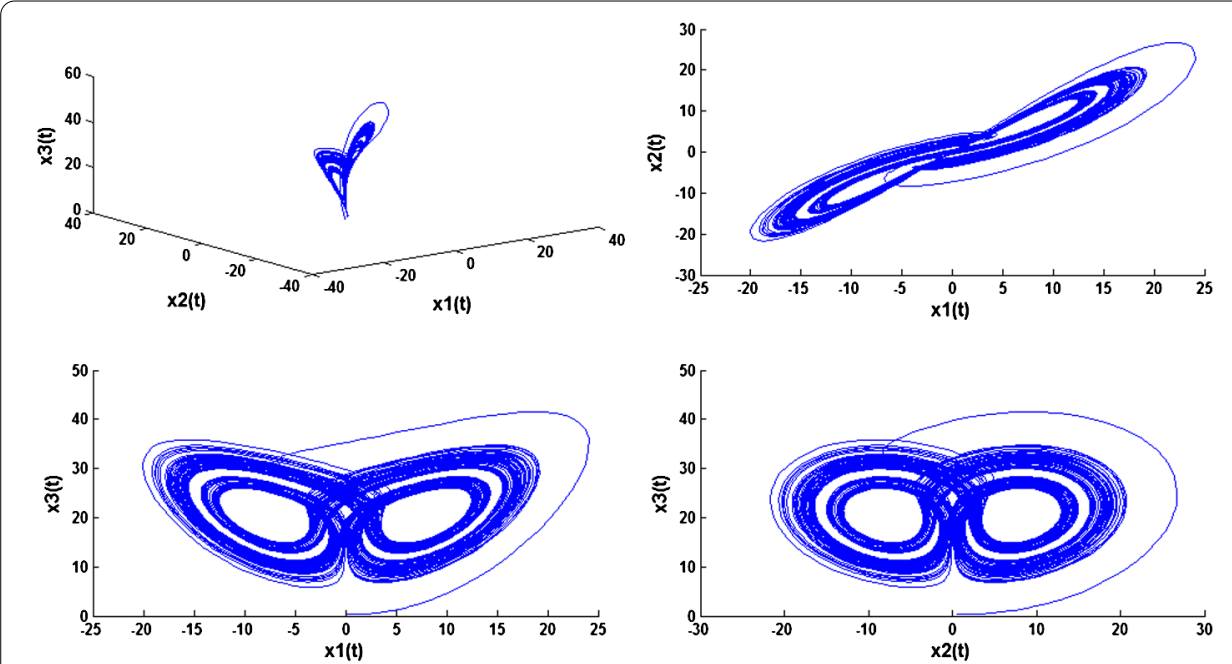

Figure 4 Different plots of the chaotic attractor of the incommensurate fractional Lü system (35).

As shown in [41], a chaotic attractor (see Figure 4) is found in the incommensurate system (35) when $(\alpha, \beta, \gamma)=(36,3,20)$ and $\left(p_{1}, p_{2}, p_{3}\right)=(0.985,0.99,0.98)$. The linear part of the master system (35) is given by

$$
A=\left(\begin{array}{ccc}
-36 & 36 & 0 \\
0 & 20 & 0 \\
0 & 0 & -3
\end{array}\right) .
$$

The incommensurate hyperchaotic fractional-order Lorenz system is selected as the slave system [42]

$$
\left\{\begin{array}{l}
D_{t}^{q_{1}} y_{1}=a\left(y_{2}-y_{1}\right)+y_{4}+u_{1}, \\
D_{t}^{q_{2}} y_{2}=c y_{1}-y_{2}-y_{1} y_{3}+u_{2}, \\
D_{t}^{q_{3}} y_{3}=-b y_{3}+y_{1} y_{2}+u_{3} \\
D_{t}^{q_{4}} y_{4}=r y_{4}+y_{2} y_{3}+u_{4} .
\end{array}\right.
$$

Note that the uncontrolled system (37) exhibits hyperchaotic behavior when

$$
\begin{aligned}
& \left(q_{1}, q_{2}, q_{3}, q_{4}\right)=(0.94,0.96,0.97,0.99) \text { and } \\
& (a, b, c, r)=\left(10, \frac{8}{3}, 28,-1\right) .
\end{aligned}
$$

Different plots of the hyperchaotic attractor are reported in Figure 5. By selecting the scaling parameters

$$
\left(\alpha_{31}, \alpha_{32}, \alpha_{33}, \alpha_{34}\right)=(2,1,4,-1)
$$

the error system between the master system (35) and the slave system (37) becomes

$$
e_{1}=x_{1}-y_{1}
$$



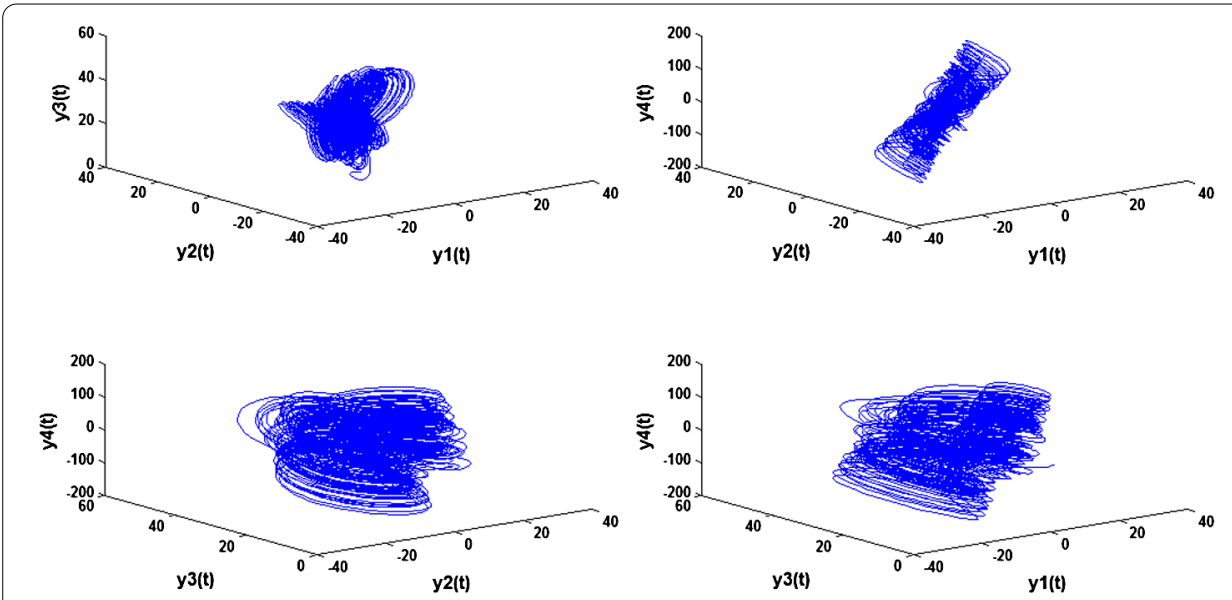

Figure 5 Different plots of the hyperchaotic attractors of the incommensurate fractional Lorenz system (37).

$$
\begin{aligned}
& e_{2}=x_{2}-\left(-y_{2}\right), \\
& e_{3}=x_{3}-\left(2 y_{1}+y_{2}+4 y_{3}-y_{4}\right) .
\end{aligned}
$$

By Theorem 4 there exists a gain matrix $L \in \mathbb{R}^{3 \times 3}$ such that $I S, A S$, and IFSHPS coexist between systems (35) and (37). For example, if the control matrix $L$ is selected as

$$
L=\left(\begin{array}{ccc}
0 & 36 & 0 \\
0 & 21 & 0 \\
0 & 0 & 0
\end{array}\right)
$$

then we can readily verify that the roots of the equation

$$
\operatorname{det}\left(\operatorname{diag}\left(\lambda^{0.94 M}, \lambda^{0.96 M}, \lambda^{0.97 M}\right)+L-A\right)=0
$$

are given by

$$
\begin{aligned}
& \lambda_{1}=36^{\frac{1}{0.94 M}}\left(\cos \frac{\pi}{0.94 M}+\mathbf{i} \sin \frac{\pi}{0.94 M}\right), \\
& \lambda_{2}=\cos \frac{\pi}{0.96 M}+\mathbf{i} \sin \frac{\pi}{0.96 M}, \\
& \lambda_{3}=3^{\frac{1}{0.97 M}}\left(\cos \frac{\pi}{0.97 M}+\mathbf{i} \sin \frac{\pi}{0.97 M}\right),
\end{aligned}
$$

where $M$ is the least common multiple of the denominators of the numbers $0.94,0.96$, and 0.97. Since this results in $\left|\arg \left(\lambda_{i}\right)\right|>\frac{\pi}{2 M}(i=1,2,3)$, all the conditions of Theorem 4 are satisfied. Thus, the controllers $u_{1}, u_{2}, u_{3}$, and $u_{4}$ can be written as

$$
\left\{\begin{array}{l}
u_{1}=36 e_{1}+D^{0.94} x_{1}-10\left(y_{2}-y_{1}\right)-y_{4} \\
u_{2}=-e_{2}-D^{0.96} x_{2}-28 y_{1}+y_{2}+y_{1} y_{3} \\
u_{3}=\frac{1}{4} e_{3}+\frac{1}{4} D^{0.97}\left(x_{3}-2 y_{1}-y_{2}+y_{4}\right)+\frac{8}{3} y_{3}-y_{1} y_{2} \\
u_{4}=0
\end{array}\right.
$$




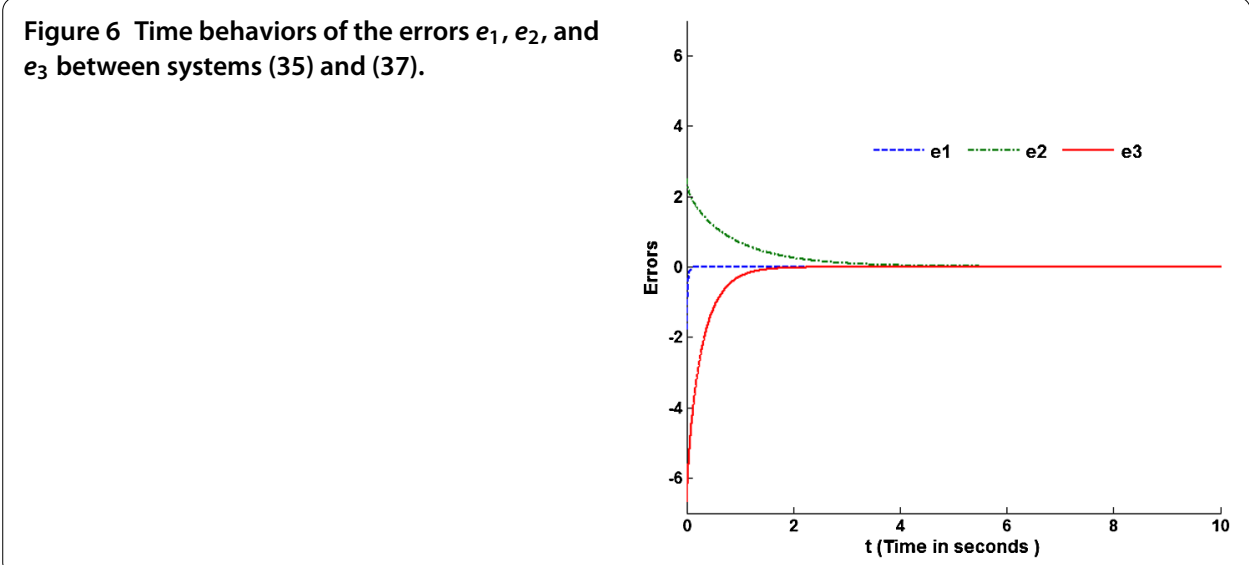

indicating that synchronization is successfully achieved between systems (35) and (37). The error system can be described as follows:

$$
\left\{\begin{array}{l}
D^{0.94} e_{1}=-36 e_{1}, \\
D^{0.96} e_{2}=-e_{2}, \\
D^{0.97} e_{3}=-3 e_{3} .
\end{array}\right.
$$

The fractional Euler integration method has been used to numerically solve systems (35) and (37) with control inputs given by (44). The initial values for the master and slave systems are $\left[x_{1}(0), x_{2}(0), x_{3}(0)\right]=[0.2,0.5,0.3]$ and $\left[y_{1}(0), y_{2}(0), y_{3}(0), y_{4}(0)\right]=[2,-2,1,-1]$, respectively. Based on (40), the initial states of the error system $(45)$ are $\left[e_{1}(0), e_{2}(0), e_{3}(0)\right]=$ $[-1.8,2.5,-6.7]$. The time behaviors of the errors $e_{i}(i=1,2,3)$ are shown in Figure 6, clearly indicating that synchronization is effectively achieved between systems (35) and (37). Specifically, by considering the errors in (40) it can be concluded that identical synchronization (IS), antiphase synchronization (AS), and inverse full state hybrid projective synchronization (IFSHPS) coexist when synchronizing the chaotic fractional-order incommensurate Lü system (35) and the hyperchaotic fractional-order incommensurate Lorenz system (37).

\section{Discussion}

The aim of this section is to highlight the novelty introduced by the present approach. First of all, it is worth analyzing the results achieved so far in the literature on the same topic, that is, the coexistence of some synchronization types in different dimensional fractional-order chaotic systems. This topic is almost unexplored in the literature [31, 32]. For example, in [31] the authors have presented a hybrid synchronization method for fractional-order systems, which is a combination of only two synchronization types, that is, generalized synchronization and inverse generalized synchronization. Our approach is more general than that in [31], since it guarantees the coexistence of three different synchronization types, that is, identical synchronization (IS), antiphase synchronization $(A S)$, and inverse full state hybrid projective synchronization (IFSHPS). Another method for fractional-order systems has been developed in [32], where a combination of full state hybrid projective synchronization (FSHPS) and inverse full state hybrid projective synchronization (IFSHPS) has been presented. However, the method in [32] can only be ap- 
plied to incommensurate systems, besides the fact that it allows the coexistence of only two synchronization types. Our approach is more general than that in [32], since it can be applied to both commensurate and incommensurate systems (see Theorems 3 and 4, respectively), besides the fact that it guarantees the coexistence of three different synchronization types (instead of only two, as in [32]).

Based on previous considerations, it should be clear that the method proposed herein provides a contribution to the topic related to the coexistence of some fractional synchronization types, since it guarantees the coexistence of three different synchronization types for both commensurate and incommensurate fractional-order systems of different dimensions and different orders. This increased complexity related to both the number of synchronization types and the capability to synchronize chaotic dynamics with hyperchaotic ones and provides a deeper insight into the synchronization phenomena between systems described by fractional differential equations.

\section{Conclusions}

In this paper, we have presented a new approach to rigorously study the coexistence of some synchronization types between fractional-order chaotic systems characterized by different dimensions and different orders. The paper has shown that identical synchronization (IS), antiphase synchronization (AS), and inverse full state hybrid projective synchronization (IFSHPS) coexist when synchronizing a three-dimensional master system with a fourth-dimensional slave system. It has been shown that the approach presents the remarkable feature of being both rigorous and applicable to a wide class of commensurate and incommensurate systems of different dimensions and orders. All the numerical examples reported through the paper have clearly highlighted the capability of the proposed approach in successfully achieving the co-existence of IS, AS, and IFSHPS between chaotic and hyperchaotic systems of different dimensions for both commensurate and incommensurate fractional-order systems. These examples of coexistence have included the chaotic commensurate three-dimensional Rössler system of order 2.7, the hyperchaotic commensurate four-dimensional Chen system of order 3.84 , the chaotic incommensurate three-dimensional Lü system of order 2.955, and the hyperchaotic incommensurate fourdimensional Lorenz system of order 3.86. Finally, we would stress that the topic related to the coexistence of synchronization types in fractional-order systems is almost unexplored in the literature. We feel that the additional features introduced by the conceived approach, related to both the number of coexisting synchronization types and the capability to synchronize chaotic dynamics with hyperchaotic ones, provides a deeper insight into the synchronization phenomena between systems described by fractional differential equations. 
Authors' contributions

$A O$ and VTP suggested the model. AO, XW, VTP, GG, and TZ helped in results interpretation and manuscript evaluation. XW, GG, and TZ helped to evaluate, revise, and edit the manuscript. XW, GG, and TZ supervised the development of work. $\mathrm{AO}$ and VTP drafted the article. All authors read and approved the final manuscript.

\section{Author details}

'Department of Mathematics, University of Larbi Tebessi, Tebessa, Algeria. ${ }^{2}$ Institute for Advanced Study, Shenzhen University, Shenzhen, China. ${ }^{3}$ Modeling Evolutionary Algorithms Simulation and Artificial Intelligent, Faculty of Electrical \& Electronics Engineering, Ton Duc Thang University, Ho Chi Minh City, Vietnam. ${ }^{4}$ Dipartimento Ingegneria Innovazione, Università del Salento, Lecce, Italy. ${ }^{5}$ Department of Material Sciences, University of Tebessa, Tebessa, Algeria.

\section{Publisher's Note}

Springer Nature remains neutral with regard to jurisdictional claims in published maps and institutional affiliations.

Received: 29 September 2017 Accepted: 12 January 2018 Published online: 23 January 2018

\section{References}

1. Carroll, TL, Pecora, LM: Synchronizing chaotic circuits. IEEE Trans. Circuits Syst. 38(4), 453-456 (1991)

2. Wen, G, Grassi, G, Feng, Z, Liu, X: Special issue on advances in nonlinear dynamics and control. J. Franklin Inst. 352(8), 2985-2986 (2015)

3. Wang, XF, Chen, GR: Synchronization in small-world dynamical networks. Int. J. Bifurc. Chaos 12, 187-192 (2002)

4. Cao, JD, Li, HX, Ho, DWC: Synchronization criteria of Lur's systems with time-delay feedback control. Chaos Solitons Fractals 23, 1285-1298 (2005)

5. He, WL, Qian, F, Cao, JD, Han, QL: Impulsive synchronization of two nonidentical chaotic systems with time-varying delay. Phys. Lett. A 375, 498-504 (2011)

6. Pourdehi, S, Karimaghaee, P, Karimipour, D: Adaptive controller design for lag-synchronization of two non-identical time-delayed chaotic systems with unknown parameters. Phys. Lett. A 375, 1769-1778 (2011)

7. Rehan, M: Synchronization and anti-synchronization of chaotic oscillators under input saturation. Appl. Math. Model. 37, 6829-6837 (2013)

8. Wu, A: Hyperchaos synchronization of memristor oscillator system via combination scheme. Adv. Differ. Equ. 2014,86 (2014)

9. Pecora, LM, Carroll, TL: Synchronization of chaotic circuits. Chaos 25, 097611 (2015)

10. Abrams, DM, Pecora, LM, Motter, AE: Introduction to focus issue - patterns of network synchronization. Chaos 26 , 094601 (2016)

11. Manieri, R, Rehacek, J: Projective synchronization in three-dimensional chaotic systems. Phys. Rev. Lett. 82(15), 3042-3045 (1999)

12. $\mathrm{Hu}, \mathrm{M}, \mathrm{Xu}, \mathrm{Z}, \mathrm{Zhang}, \mathrm{R}$ : Full state hybrid projective synchronization in continuous-time chaotic (hyperchaotic) systems. Commun. Nonlinear Sci. Numer. Simul. 13(2), 456-464 (2008)

13. Ouannas, A, Grassi, G: Inverse full state hybrid projective synchronization for chaotic maps with different dimensions. Chin. Phys. B 25(9), 090503 (2016)

14. Ouannas, A, Azar, AT, Ziar, T: On inverse full state hybrid function projective synchronization for continuous-time chaotic dynamical systems with arbitrary dimensions. Differ. Equ. Dyn. Syst. (2017) https://doi.org/10.1007/s12591-017-0362-x

15. Ouannas, A, Grassi, G: A new approach to study the coexistence of some synchronization types between chaotic maps with different dimensions. Nonlinear Dyn. 86(2), 1319-1328 (2016)

16. Ouannas, A: Co-existence of various synchronization-types in hyperchaotic maps. Nonlinear Dyn. Syst. Theory 16(3), 312-321 (2016)

17. Ouannas, A, Azar, AT, Vaidyanathan, S: New hybrid synchronization schemes based on coexistence of various types of synchronization between master-slave hyperchaotic systems. Int. J. Comput. Appl. Technol. 55(2), 112-120 (2017)

18. Pinto, CMA: Strange dynamics in a fractional derivative of complex-order network of chaotic oscillators. Int. J. Bifurc Chaos 25(1), $1550003(2015)$

19. Ugurlu, E, Baleanu, D, Tas, K: Regular fractional differential equations in the Sobolev space. Fract. Calc. Appl. Anal. (2017). https://doi.org/10.1515/fca-2017-0041

20. Cafagna, D, Grassi, G: On the simplest fractional-order memristor-based chaotic system. Nonlinear Dyn. 70(2), 1185-1197 (2012)

21. Cafagna, D, Grassi, G: Fractional-order systems without equilibria: the first example of hyperchaos and its application to synchronization. Chin. Phys. B 24(8), 080502 (2015)

22. Grigorenko, I, Grigorenko, E: Chaotic dynamics of the fractional Lorenz system. Phys. Rev. Lett. 91, 034101 (2003)

23. Li, CP, Deng, WH, Xu, D: Chaos synchronization of the Chua system with a fractional order. Physica A 360, 171-185 (2006)

24. Li, C, Chen, G: Chaos and hyperchaos in fractional order Rössler equations. Physica A 341, 55-61 (2004)

25. Lu, JG, Chen, G: A note on the fractional-order Chen system. Chaos Solitons Fractals 27, 685-688 (2006)

26. Deng, WH, Li, CP: Chaos synchronization of the fractional Lü system. Physica A 353, 61-72 (2005)

27. Wu, GC, Baleanu, D, Huang, LL: Chaos synchronization of the fractional Rucklidge system based on new Adomian polynomials. J. Appl. Nonlinear Dyn. (2017). https://doi.org/10.5890/JAND.2017.09.006

28. Ouannas, A, Al-sawalha, MM, Ziar, T: Fractional chaos synchronization schemes for different dimensional systems with non-identical fractional-orders via two scaling matrices. Optik 127(20), 8410-8418 (2016)

29. Ouannas, A, Grassi, G, Ziar, T, Odibat, Z: On a function projective synchronization scheme for non-identical fractional-order chaotic (hyperchaotic) systems with different dimensions and orders. Optik 136, 513-523 (2017)

30. Ouannas, A, Abdelmalek, S, Bendoukha, S: Coexistence of some chaos synchronization types in fractional-order differential equations. Electron. J. Differ. Equ. 2017, 128 (2017) 
31. Ouannas, A, Azar, AT, Vaidyanathan, S: A robust method for new fractional hybrid chaos synchronization. Math. Methods Appl. Sci. 40(5), 1804-1812 (2017)

32. Ouannas, A, Azar, AT, Vaidyanathan, S: A new fractional hybrid chaos synchronization. Int. J. Model. Identif. Control 27(4), 314-323 (2017)

33. Caputo, M: Linear models of dissipation whose $q$ is almost frequency independent. Geophys. J. R. Astron. Soc. 13, 529-539 (1967)

34. Oldham, KB, Spanier, J: The Fractional Calculus. Academic Press, New York (1974)

35. Podlubny, I: Fractional Differential Equations. Academic Press, New York (1999)

36. Samko, SG, Klibas, AA, Marichev, Ol: Fractional Integrals and Derivatives: Theory and Applications. Gordon \& Breach, Amsterdam (1993)

37. Matignon, D: Stability results of fractional differential equations with applications to control processing. IMACS, IEEE-SMC, Lille, France (1996)

38. Gorenko, R, Mainardi, F: Fractional calculus: integral and differential equations of fractional order. In: Carpinteri, A, Mainardi, F (eds.) Fractals and Fractional Calculus in Continuum Mechanics. Springer, New York (1997)

39. Li, Y, Chen, Y, Podlubny, I: Stability of fractional-order nonlinear dynamic systems: Lyapunov direct method and generalized Mittag-Leffler stability. Comput. Math. Appl. 59, 1810-1821 (2010)

40. Chen, D, Zhang, R, Liu, X, Ma, X: Fractional order Lyapunov stability theorem and its applications in synchronization of complex dynamical networks. Commun. Nonlinear Sci. Numer. Simul. 19, 4105-4121 (2014)

41. Li, C, Chen, G: Chaos and hyperchaos in fractional order Rössler equations. Physica A 341, 55-61 (2004)

42. Li, T-Z, Wang, Y, Luo, M-K: Control of fractional chaotic and hyperchaotic systems based on a fractional order controller. Chin. Phys. B 23(8), 080501 (2014)

43. Deng, W, Li, C, Lü, J: Stability analysis of linear fractional differential system with multiple time delays. Nonlinear Dyn 48, 409-416 (2007)

\section{Submit your manuscript to a SpringerOpen ${ }^{\circ}$ journal and benefit from:}

- Convenient online submission

- Rigorous peer review

- Open access: articles freely available online

- High visibility within the field

- Retaining the copyright to your article

Submit your next manuscript at $\gg$ springeropen.com 